Comment on "Influence of Pair Breaking and Phase Fluctuations on Disordered High $T_{c}$ Cuprate Superconductors"

In a recent Letter 1], Rullier-Albenque et al. studied the $T_{c}$ degradation under electron irradiation of Y-123 single crystals. They have measured the in-plane resistivity $\rho_{a b}(T)$ in a broad range of defect contents $x_{d}$, the value of $x_{d}$ being proportional to $\Delta \rho_{a b}$, the increase in $\rho_{a b}$ upon irradiation. It was found that $T_{c}$ unexpectedly decreased quasilinearly with $x_{d}$ in the whole range from $T_{c 0}$ down to $T_{c}=0$. The authors of Ref. [1] arrived at a conclusion that experimental data are at variance with Abrikosov-Gor'kov (AG) pair breaking theory 2 and point to a significant role of phase fluctuations 3 of the order parameter $\Delta(\mathbf{p})$ in high- $T_{c}$ cuprates. In this Comment, we show that the data reported in Ref. 1] are in fact not inconsistent with the pair-breaking theories if (i) the deviation from pure $d$-wave symmetry of $\Delta(\mathbf{p})$ and (ii) the existence of magnetic scatterers in irradiated samples are properly taken into account.

The authors of Ref. 1] made use of the AG formula 2] for $d$-wave superconductors, $\ln \left(T_{c 0} / T_{c}\right)=\Psi(1 / 2+$ $\left.1 / 2 \pi T_{c} \tau\right)-\Psi(1 / 2)$, where $\tau$ is the electron scattering time, $\tau^{-1} \propto x_{d} \propto \Delta \rho_{a b}$. This formula gives a downward curvature of $T_{c}\left(\Delta \rho_{a b}\right)$ curve, contrary to experimental observations [1]. Note, however, that the symmetry of $\Delta(\mathbf{p})$ in high- $T_{c}$ cuprates may be different from pure $d$-wave [4, 5]. Besides, irradiation may result in appearance of magnetic scatterers along with nonmagnetic ones since radiation defects disturb antiferromagnetic correlations between copper spins. The AG-like formula that accounts for both those effects [6] reads $\ln \left(T_{c 0} / T_{c}\right)=(1-\chi)\left[\Psi\left(1 / 2+1 / 2 \pi T_{c} \tau_{m}\right)-\Psi(1 / 2)\right]+$ $\chi\left[\Psi\left(1 / 2+1 / 4 \pi T_{c} \tau_{n}+1 / 4 \pi T_{c} \tau_{m}\right)-\Psi(1 / 2)\right]$, where $\tau_{n}$ and $\tau_{m}$ are scattering times due to nonmagnetic and magnetic defects, respectively, the coefficient $\chi=1-$ $\langle\Delta(\mathbf{p})\rangle_{F S}^{2} /\left\langle\Delta^{2}(\mathbf{p})\right\rangle_{F S}$ is a measure of $\Delta(\mathbf{p})$ anisotropy on the Fermi surface $(\chi=1$ for $d$-wave, $0<\chi<1$ for mixed $(d+s)$-wave or anisotropic $s$-wave, $\chi=0$ for isotropic $s$-wave).

An account for combined effect of both nonmagnetic and magnetic scatterers on $T_{c}$ and/or an assumption about a non-pure $d$-wave $\Delta(\mathbf{p})$ allows for a quantitative explanation of the experimental data within the AG-like pair breaking theory, without resorting to phase fluctuations effects. Fig. 1 shows the measured $T_{c} / T_{c 0}$ versus $\Delta \rho_{a b}$ taken from Ref. [1] along with the curves computed for $\chi=0.9$ and various values of the coefficient $\alpha=\tau_{m}^{-1} /\left(\tau_{n}^{-1}+\tau_{m}^{-1}\right)$ that specifies the relative contribution to the total scattering rate from magnetic scatterers 6]. Here we make use of the relation [6] $\tau_{n}^{-1}+\tau_{m}^{-1}=\left(\omega_{p l}^{2} / 4 \pi\right) \Delta \rho_{a b}$, where $\omega_{p l}$ is a characteristic energy which should not necessarily coincide with the plasma frequency determined by, e. g., optical spectroscopy. The quasilinear dependence of $T_{c}$ on $\Delta \rho_{a b}$ in $\mathrm{YBa}_{2} \mathrm{Cu}_{3} \mathrm{O}_{7}$ is quantitatively reproduced at $\omega_{p l}=0.75$
$\mathrm{eV}$ and $\alpha=0 \div 0.01$. This value of $\omega_{p l}$ is a factor of 1.4 different from directly measured values of the plasma frequency in Y-123. Although our choice of $\omega_{p l}$ is, to some extent, arbitrary, the change in $\omega_{p l}$ will result just in the change of the best fitting values of $\chi$ and $\alpha$, e. g., $\chi \approx 0.8$ and $0.6, \alpha=0.04 \pm 0.02$ and $0.04 \pm 0.01$ at $\omega_{p l}=0.8$ and $1 \mathrm{eV}$, respectively. The data for $\mathrm{YBa}_{2} \mathrm{Cu}_{3} \mathrm{O}_{6.6}$ can also be well fitted within this approach.

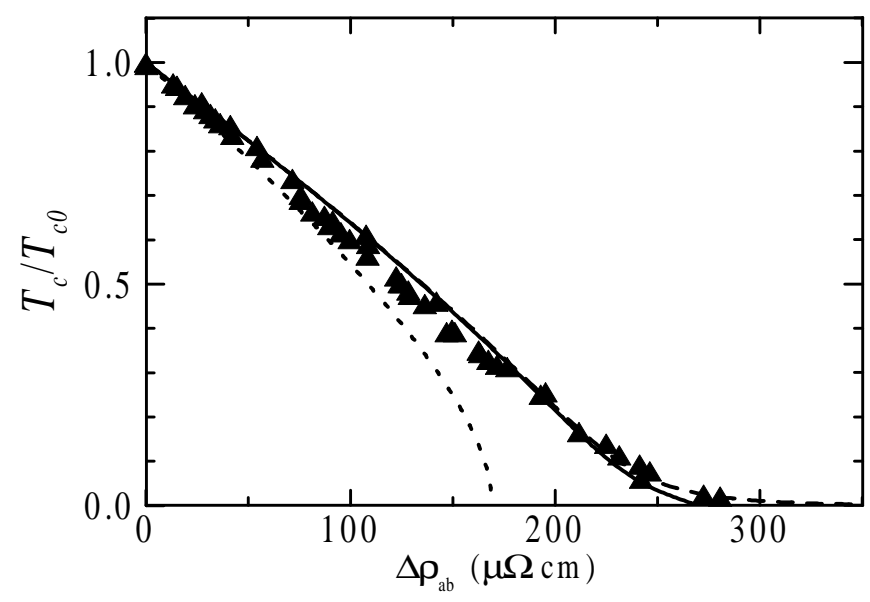

FIG. 1: $T_{c} / T_{c 0}$ versus $\Delta \rho_{a b}$ in electron irradiated $\mathrm{YBa}_{2} \mathrm{Cu}_{3} \mathrm{O}_{7}$ crystals. Experiment [1] (triangles). Theory [6] for $\omega_{p l}=0.75$ $\mathrm{eV}, \chi=0.9$ and $\alpha=0$ (dashed line), 0.01 (solid line), and 1 (dotted line).

Finally, the arguments presented in Ref. 1] concerning the upward curvature of $T_{c}\left(\Delta \rho_{a b}\right)$ curve required to explain the maximum of the transition width $\delta T_{c}$ as a function of $\Delta \rho_{a b}$ seem to be incompatible with experimental data since the curvature of the measured $T_{c}\left(\Delta \rho_{a b}\right)$ dependence is close to zero in the whole range of $\Delta \rho_{a b}$.

I am grateful to A. V. Kuznetsov for assistance. The work was supported by the Russian Ministry of Industry, Science, and Technology, Grant No 40.012.1.1.1357.

\section{A. Openov}

Moscow Engineering Physics Institute, Moscow 115409, Russia.

PACS numbers: 74.62.Dh, 74.20.-z, 74.25.Fy, 74.72.Bk

[1] F. Rullier-Albenque et al., Phys. Rev. Lett. 91, 047001 (2003).

[2] A. A. Abrikosov and L. P. Gor'kov, Sov. Phys. JETP 12, 1243 (1961).

[3] V. J. Emery and S. A. Kivelson, Nature 374, 434 (1995).

[4] B. H. Brandow, Phys. Rev. B 65, 054503 (2002).

[5] G. M. Zhao, Phys. Rev. B 64, 024503 (2001); cond-mat/0305483

[6] L. A. Openov, Phys. Rev. B 58, 9468 (1998). 